CERN-TH. 7429/94

\title{
HIGH DENSITY QCD AND ENTROPY PRODUCTION AT HEAVY ION COLLIDERS
}

\author{
K. Geiger \\ CERN TH-Division, CH-1211 Geneva 23, Switzerland
}

\begin{abstract}
The role of entropy production in the context of probing QCD properties at high densities and finite temperatures in ultra-relativistic collisions of heavy nuclei is inspected. It is argued that the entropy generated in these reactions provides a powerful tool to investigate the space-time evolution and the question whether and how a deconfined plasma of quarks and gluons is formed. I will address the questions how entropy is produced, and how it is measurable. The uncertainties in predicting the different contributions to the total entropy and particle multiplicities during the course of heavy ion collisions are also discussed.
\end{abstract}

e-mail: klaus@surya11.cern.ch

CERN-TH. 7429/94, September 1994

\footnotetext{
${ }^{1}$ Talk presented at the NATO Advanced Research Workshop on Hot Hadronic Matter: Theory and Experiment, Divonne, France, July 1994.
} 


\section{INTRODUCTION}

In this talk I would like to present a perspective of what we can learn about aspects of QCD at high density with the advent of a new generation of accelerators. Particularly the future heavy ion $(A A)$ colliders, the BNL Relativistic Heavy Ion Collider (RHIC) with maximum available beam energy $\sqrt{s}=200 \mathrm{~A} \mathrm{GeV}$ (gold on gold) and the CERN Large Hadron Collider (LHC) with $\sqrt{s}=5500 A \mathrm{GeV}$ (lead on lead), will provide for the first time the opportunity to study nuclear matter under extreme density compression and very high temperatures, and possibly the formation of a deconfined quark-gluon plasma (QGP). A major goal of the experimental programs at RHIC and LHC is to explore new phenomena associated with the dynamics of quarks and gluons in the hot, ultra-dense environment that is created in these collisions, including the expected (non-) equilibrium QCD phase transition.

\section{THE REGIME OF HIGH DENSITY QCD}

Fig. 1 shows the map of QCD as it is understood today. It exhibits three very distinct regions of QCD with quite different physics [1]. On the horizontal axis, $r$ characterizes the space-time distance that can be resolved by probing QCD properties in certain dynamical processes. For instance, when probing a nucleon or nucleus in a deep inelastic scattering event with a momentum transfer $Q^{2}$, the photon acts as a microscope with a resolution $r \sim 1 / Q$. On the vertical axis, the density $\rho_{q g}$ is the number of quark and gluon quanta with a definite value of rapidity $y=\ln (1 / x)$ that is seen by our probe in the transverse plane,

$$
\rho_{q g}=\frac{1}{\pi R^{2}} \frac{d N_{q g}}{d y} \simeq \frac{A x f\left(x, Q^{2}\right)}{\pi R^{2}},
$$

where $f\left(x, Q^{2}\right)$ denotes the sum of quark and gluon structure functions, $R$ is the nucleon radius and $A$ is the number of nucleons.

The three regions of Fig. 1 are:

(i) $r \ll 1 \mathrm{fm}$ and $\rho \ll R^{-2}$ : This is the small distance, low density regime, where the powerful methods of perturbative QCD (pQCD) apply. 
(ii) $r \approx 1 \mathrm{fm}:$ This is the non-perturbative QCD (npQCD) domain, where one has to deal with the complex mechanisms of confinement and relys on lattice calculations and QCD sum rules.

(iii) $r \ll 1 \mathrm{fm}$ and $\rho \gtrsim R^{-2}$ : Here we can probe a high density of partons at short distances (hdQCD). Perturbative techniques can be applied, but in addition, one must tackle important density effects that lead to interference and collective phenomena.

The hdQCD regime is the exciting new field that opens up with the experimental programs at HERA, RHIC and LHC. There are two ways to obtain a system of partons with large density: One way to penetrate hdQCD is deep inelastic ep scattering $(A=1)$ at high energy in the region of very small Bjorken $x \ll 1$. For instance, at HERA, the new experiments measure 30-50(!) gluons in a proton at $x=10^{-4}$ [2]. The other access to the hdQCD region is through collisions of heavy nuclei, in which one can reach high parton densities at not so very high energies or small $x$, due to the large number of overlapping nucleons $(A \gg 1)$. This presumably can be achieved at RHIC $\left(x \approx 10^{-1}\right)$, but certainly at LHC $\left(x \approx 10^{-3}\right)$. In particular at LHC both conditions, small $x$ and large $A$, are combined.

\section{THE ROLE OF ENTROPY IN HEAVY ION COLLISIONS}

For the remainder of this talk, I will focus on the hdQCD physics in heavy ion collisions at RHIC and LHC. Qualitatively, the expected space-time evolution of these reactions can be summarized as illustrated in Fig. 2 [6]: (1) Immediately after the first nuclear contact, the partons of the colliding nuclei begin to interact frequently with each other, resulting in a vehement materialization of excited quanta. (2) The excited partons can rescatter and emit new particles and thereby evolve through a pre-equilibrium stage towards a thermalized quark gluon plasma state, from which the system evolves further according to the laws of relativistic hydrodynamics. (3) The plasma expands and cools, and eventually a phase transition - perhaps via a mixed parton-hadron phase - into a purely hadronic gas occurs.

(4) The freeze-out of this excited hadron matter produces the final hadron yield.

One of the most valuable quantities for extracting information about the dynamical 
evolution is the production of entropy. With each of the above stages (1)-(4) a certain amount of generated entropy is associated. The entropy is generally defined in terms of the density matrix $\hat{\rho}$ of the quantum system, $S=-\operatorname{Tr} \hat{\rho} \ln \hat{\rho}$ [3], or equivalently, in terms of the particle distribution (Wigner) function $W(r, p)$, where $r \equiv r^{\mu}=(t, \vec{r}), p \equiv p^{\mu}=(E, \vec{p})$ :

$$
S(t)=-\int \frac{d^{3} r d^{3} p}{(2 \pi)^{3}} W(r, p) \ln W(r, p),
$$

(neglecting Fermi or Bose statistics). Thus $S$ contains valuable information about the time evolution of the system in full phase-space, with the complete history up to time $t$ embodied in the function $W$. Measuring the entropy as a function of time would therefore

allow to trace the space-time evolution of a nuclear collision. Furthermore the rate of entropy production reflects the degree and time scale of equilibration, since $d S / d t \rightarrow 0$ as an equilibrium state is reached [4].

Clearly, it is not easy to extract the time evolution of $S$ from experiment. First of all the entropy is not directly observable, it can be measured only indirectly through the multiplicities of produced particles in a reaction and the amount of transverse energy generated. Secondly, to measure the rate of entropy or particle production, one has to identify signals of characteristic particle emission from different stages of a nuclear collision. Good probes of time rate of change are dileptons, photons, strange and charm production [5, 6]. To this end, let me pose the following two questions:

How is entropy produced in heavy ion collisions at RHIC and LHC?

How can we measure entropy in these reactions?

\section{HOW IS ENTROPY PRODUCED?}

Most of the entropy and transverse energy is expected to be produced already within the first $f m$ after nuclear contact by very frequent so-called semihard parton interactions with only a few $\mathrm{GeV}$ momentum transfer $\llbracket 7$, 8]. This corresponds to the pre-equilibrium regime in Fig. 2. Once the system has reached a thermal equilibrium state, the entropy production vanishes in space-time, and ideally the final transition from the quark-gluon phase to the hadron phase and its freeze out should keep the entropy constant [9]. Let me 
write the total produced entropy that is produced during the course of a heavy ion collision as

$$
\Delta S=\Delta S_{\text {prim }}^{(q g)}+\Delta S_{\text {sec }}^{(q g)}+\Delta S^{(h a d)}
$$

The three contributions arise as follows:

(i) $\Delta S_{\text {prim }}^{(q g)}$, large: primary parton production due to the materialization of virtual "preexisting" quanta in the colliding nuclei that are set free by very frequent initial parton scatterings.

(ii) $\Delta S_{\text {sec }}^{(q g)}$, very large: secondary parton production due to intense gluon bremsstrahlung off partons that have been excited in scatterings, plus production by rescatterings which become increasingly probable as the parton density grows.

(ii) $\Delta S^{(\text {had) }}$, small: entropy produced through the parton-hadron transition (which ideally should be neglegible) plus additional entropy generated by decay of formed "prehadrons" and hadronic resonances into the final stable hadron states.

As an illustrative example, Fig. 3a displays the time development of the specific entropy $(S / N)(t)$ arising from $\Delta S_{\text {prim }}^{(q g)}$ and $\Delta S_{\text {sec }}^{(q g)}$ as calculated within the parton cascade model [6] for various collider energies. The curves show a rapid build-up of $S / N$ and relax approximately exponential to reach their final values between 3.9 and 4.3. Comparing these values with $(S / N)_{\text {ideal }} \simeq 4$ for an ideal gas of non-interacting massless quarks and gluons, one sees that the difference between the resulting entropy of the realistic model calculation and the idealized case amounts less than $10 \%$. Although the model includes massive quarks and accounts for interactions among the partons, the system of partons behaves effectively like an almost ideal gas. Fig. 3b shows the corresponding relaxation times versus $\sqrt{s} / A$, which are evidently very short and indicate a very rapid thermalization (plasma formation) within less than $0.5 \mathrm{fm}$.

\section{HOW CAN ENTROPY BE MEASURED?}

As mentioned above, the entropy ideally must stay constant once the system has reached an equilibrium (QGP) state. In this context, the only effect of the parton-hadron phase 
transition is a reorganization of the degrees of freedom from the colored quarks and gluons to the color singlet hadrons, mostly pions. Now, experiments of deep inelastic ep-scattering, $e^{+} e^{-}$-annihilation, Drell Yan, etc., strongly support the hypothesis of local parton-hadron duality [10]. That is, it appears that in high energy processes the mechanism of hadronization is a universal and local phenomenon, independent of the partons prehistory. The measured hadron multiplicity turns out to be simply equal the calculated parton multiplicity times a constant (e.g. $\left.N^{(\pi)}=1.1 N^{(q g)}\right)$.

On the other hand, if there is a first order QCD phase transition at a temperature $T_{c}$, the ratio of the entropy density in the pion plasma, $s^{(\pi)}$, to that of the quark-gluon plasma, $s^{(q g)}$, can be estimated from the effective number of degrees of freedom in the two phases at $T_{c}$ as $r=s^{(\pi)} / s^{(q g)} \approx 0.7$ [9]. Under these assumptions for zero impact parameter collisions the total produced entropy can be measured by

$$
\frac{d S}{d y}=c^{(q g)}\left(\frac{d N^{(q g)}}{d y}\right)_{b=0} \approx \frac{c^{(\pi)}}{r}\left(\frac{d N^{(\pi)}}{d y}\right)_{b=0} .
$$

where $c^{(q g)} \simeq 4$ and $c^{(\pi)}=3.6$. Thus, again one has $N^{(\pi)}=c^{(q g)} / c^{(\pi)} N^{(q g)}=1.1 N^{(q g)}$. Using the above value for $r$, one gets a relation between the entropy produced per unit rapidity and the multiplicity of final pions [11]:

$$
\left(\frac{d N_{\pi}}{d y}\right)_{b=0} \simeq 0.2 \frac{d S}{d y}
$$

The total produced entropy contained in an interval $d y$ around $y=0$ is effectively related to the observable pion multiplicity $d N^{(\pi)} / d y$. Therefore the multiplicity of pions reflects the entropy produced by the partons in the central collision region, provided that the entropy production associated with the freeze out of the excited hadron matter after the phase transition and the decay chain through resonance states is marginal. This is illustrated in Fig. 4.

\section{UNCERTAINTIES}

There are essentially two sources of uncertainties in predicting the amount of entropy and global observables as particle multiplicity or transverse energy:

a) The initial parton distributions in nucleus-nucleus collisions are subject to large uncertainty. Particularly the small $x$ behaviour of the gluon structure functions and the 
question of gluon shadowing [12] in nuclei are of primary importance, since the amount of soft gluons in the initial state sensitively determines the starting conditions for the evolution and of the general reaction dynamics [13]. These gluons densely populate phase-space and due to the large global semihard cross-section they only need to be slightly "tickled" in order to materialize. Thus the gluon content of the nuclei is a large entropy reservoir that is immediately set free because the quantum coherence is so easily destroyed [3]. Even though the recent measurements at HERA [2] of the nucleon structure functions at very small values of $x$ with an extracted gluon component $x g(x) \propto 1 / \sqrt{x}$ provide new information, the question of the corresponding form of the structure functions of nuclei composed of many nucleons requires much further investigation. Moreover, the measured structure functions, which contain solely the single particle aspect of the parton distributions, do not tell us anything about the spatial distribution of partons, or correlations among them issues which presumably play an important role for large nuclei. Fig. 5 shows the impact of structure function dependence on the parton production cross-section in $A u+A u$ collisions at RHIC and LHC energies versus momentum cut-off $p_{0}$ [13]. Most parton scatterings are semi-hard processes with momentum transfers $\simeq 2-3 \mathrm{GeV}$, thus the dominant region for particle production is at low $p_{0}$. The variation with the choice of structure functions is especially drastic at LHC, where it reaches up to an order of magnitude difference in the cross-section.

b) The other source of substantial uncertainty originates from the current lack of better knowledge of the impact of nuclear and dense medium effects which are absent in e.g. hadronic collisions, but become important for heavy ion collisions. The new physics associated with the nuclear and medium effects on the parton level [14] that determine the dynamics of $A A$ collisions drastically evident in Fig. 6. where the various curves a)-e) exhibit the characteristics of different evolution scenarios of simulations of gold on gold collisions [15], namely, a) 'naive evolution', b) plus nuclear shadowing, c) plus parton fusion and absorption, d) plus Landau-Pomeranchuk-Migdal effect, e) plus soft gluon interference. Shown are the charged particle distributions in pseudorapidity $(\eta)$, calculated for central $A u+A u$ collisions with $\sqrt{s}=200 \mathrm{~A} \mathrm{GeV}$ and $\sqrt{s}=6300 \mathrm{~A} \mathrm{GeV}$. Evidently, the particle 
density in the central rapidity region is reduced successively from the 'naive' calculation (case a) to the default result (case e) by more than a factor of 3 .

\section{SUMMARY}

Much experimental and theoretical study is required before robust conclusions for experimental observables and open theoretical questions can be drawn - although I believe, already at this point a clear understanding of the fundamental problems on the basis of perturbative QCD and transport theory has been reached [6]. Foremost, we must learn how to utilize medium effects in high density QCD, so that truly quantitative calculations become possible. Almost as importantly, we must develop a microscopic theory for the hadronization of a quark-gluon plasma that is based on QCD, doing away with the presently employed QCD-inspired models that have little predictive power in the new high density regime. If these two goals can be met, quantitative ab-initio calculations of ultrarelativistic heavy ion collisions will become feasible by the time when RHIC and LHC start operation.

\section{References}

[1] E. Levin, preprint FERMILAB-CONF-94/068-T (1994).

[2] For recent experimental and phenomenological progress, see e.g.: G. Wolf, in the proceedings of the International Workshop on Deep Inelastic Scattering, Eilat, Israel, 1994.

[3] H.-T. Elze, preprint CERN-TH 7131/93 (1994).

[4] S. de Groot, W. A. van Leuwen and C. G. van Weert, Relativistic Kinetic Theory, North Holland, Amsterdam 1980.

[5] Quark Matter '93, Proceedings of the Tenth International Conference on Ultrarelativistic Nucleus-Nucleus Collisions, Borlänge, Sweden, 1993, edited by H. A. Gustafsson et al. [Nucl. Phys. A566, 1c (1994)]. 
[6] K. Geiger, report CERN-TH 7313/94 (1994).

[7] L. V. Gribov, E. M. Levin, and M. G. Ryskin, Phys. Rep. 100, 1 (1983); E. M. Levin and M. G. Ryskin, Phys. Rep. 189, 267 (1990).

[8] K. Geiger, Nucl. Phys. A 566, 257c (1994).

[9] J. D. Bjorken, Phys. Rev. D27, 140 (1983).

[10] Ya. I. Azimov, Yu. L. Dokshitzer, V. A. Khoze and S. I. Troyan, Z. Phys. C27, 65 (1985).

[11] K. Geiger, Phys. Rev. D46, 4986 (1992).

[12] A. H. Mueller and J. Qui, Nucl. Phys. B268, 427 (1986); K. J. Eskola, J. Qui and X.-N. Wang, Phys. Rev. Lett. 72, 36 (1994). .

[13] K. Eskola, K. Kajantie and P. V. Ruuskanen, preprint HU-TFT-94-6, Helsinki 1994.

[14] See e.g.: Proceedings of the Workshop on Pre-equilibrium Parton Dynamics in Heavy Ion Collisions, edited by X. N. Wang, LBL-Report 34831, Berkeley 1993.

[15] K. Geiger, Phys. Rev. D47, 133 (1993). 


\section{FIGURE CAPTIONS}

Figure 1: The map of QCD with $\rho_{q g}$ denoting the densities of partons in the transverse plane and $r$ the distance resolved in an experiment.

Figure 2: Space-time diagram of the (longitudinal) evolution of a relativistic nucleusnucleus collision.

Figure 3: Results of a Monte Carlo simulation of gold on gold collisions at different beam energies. a) Entropy per secondary particle produced as a function of real time. b) Corresponding relaxation times versus beam energy.

Figure 4: Schematics of the entropy production in the parton and hadron phases during the evolution of nucleus-nucleus collisions.

Figure 5: Estimated parton production cross-section in heavy ion collisions at RHIC and LHC for differents sets of structure function parametrizations (from Ref. [13]).

Figure 6: Impact of nuclear and dense matter effects on a) the parton evolution and b) the resulting charged particle spectra in $A u+A u$ collisions at RHIC and LHC energies. The different evolution scenarios are explained in the text. 
This figure "fig1-1.png" is available in "png" format from: http://arxiv.org/ps/hep-ph/9409219v1 
This figure "fig2-1.png" is available in "png" format from: http://arxiv.org/ps/hep-ph/9409219v1 
This figure "fig3-1.png" is available in "png" format from: http://arxiv.org/ps/hep-ph/9409219v1 
This figure "fig1-2.png" is available in "png" format from: http://arxiv.org/ps/hep-ph/9409219v1 
This figure "fig2-2.png" is available in "png" format from: http://arxiv.org/ps/hep-ph/9409219v1 
This figure "fig1-3.png" is available in "png" format from: http://arxiv.org/ps/hep-ph/9409219v1 